\title{
Comparison of Fixative Properties of Honey with Ethanol in Oral Cytological Smears
}

\author{
Mahmood Nawaz Khan*, Ratnakar M Potekar, Savitri M Nerune and Anil K Reddy \\ Shri B.M Patil Medical College, Hospital and Research Centre. B.L.D.E University, Karnataka, India
}

\section{ABSTRACT}

Background: Fixation is an important step in cytopathological diagnosis. Ethanol is traditionally a popular and widely used fixative for cytopathological diagnosis. But ethanol is expensive and subjected to pilferage thus decreasing its ability. Ethanol denatures proteins and glycogen by precipitation. Hence in a search of better, ecofriendly and cost effective fixative, honey can be as efficient as ethanol in cytological fixation.Properties of honey such as high osmolarity, low $\mathrm{pH}$ and the presence of components such as ascorbic acid, hydrogen peroxide and phenol inhibine, all contribute to its anti-oxidative and antibacterial effects.

Methods: A cross sectional comparative study was carried out after ethical approval on healthy patients fulfilling the inclusion criteria referred to the Department of Pathology in BLDEU'S Shri B.M. Patil Medical College, Hospital and Research centre, Vijayapur. After informed consent two buccal smears were obtained from each individual, one was fixed in Ethanol and other was fixed in Honey (20\%) for a minimum of $15 \mathrm{~min}$. After staining, smears were scored for cytomorphological characters.

Results: Out of the 200 cases studied, honey fixed smears showed cytomorphological features similar to ethanol among which nuclear staining, clarity and uniformity of staining showed significant $p$ value $(p<0.05)$ where as cytoplasmic staining, cell morphology showed no statistical difference. $(\mathrm{p}>0.05)$

Conclusion: The present study offers an innovative proposal of using natural eco-friendly sweeteners, as fixative in cytopathology. The results are promising and invoke extensive large multicentric collaborative work to reach a global consensus on this fixative.

Keywords: Honey, Fixative, Oral Cytology.

\section{Introduction}

Cytopathology in the present era is a valid and wellaccepted diagnostic tool. Diagnostic accuracy always depends upon the procuring samples, fixation, staining, screening and interpretation of the specimen and quality control. Each of these steps play a vital role in diagnosis. ${ }^{[1]}$ Adequate fixation is required for proper examination of tissue or cells understudy, to reach a proper diagnosis. An ideal fixative which can fix various tissues including lymphoid, neural, muscle and fatty tissue has not been identified till date. Ideal fixative must be nontoxic, cheap and easily available, should preserve tissue for long time and should be compatible with immunohistochemical and molecular techniques. Ethanol is a well known and widely accepted fixative in Cytopathology providing excellent preservation of morphology and cellular details which are the basic requirement to make cytological diagnosis. Ethanol being an alcohol fixative preserves the tissue antigens and decreases the turnaround time and cost which are required during antigen retrieval. ${ }^{[2]}$

Ethanol though an efficient cytological fixative has few disadvantages such as it is subjected to pilferage, expensive, flammable, evaporates easily and not freely available.
It usually causes skin and eye irritation. ${ }^{[3]}$ In search of eco-friendly and ideal fixative many natural sweeteners are being experimented, among which honey has given promising results. Many studies have proved its efficacy in histopathology. It is produced from many floral sources and contains carbohydrates, vitamins, minerals, and several trace elements. Honey has inherent antibacterial, anti-oxidative properties due to high osmolarity, low $\mathrm{pH}$ and the presence of components such as ascorbic acid, hydrogen peroxide and phenol inhibine. ${ }^{[4]}$ Probable mechanism of fixation is due to presence of carbohydrates such as fructose which causes breakdown of aldehyde in presence of low $\mathrm{pH}$. These aldehydes then cross-link with tissue amino acids which leads to tissue fixation. ${ }^{[5]}$ Hence, considering this honey has also been experimented as fixative in cytology which has provided excellent cellular preservation and dehydration which are required for fixing the smears in Cytopathology.

\section{Material and methods}

A cross sectional comparative study was carried out on healthy patients fulfilling the inclusion criteria referred to the Department of Pathology in BLDEU'S Shri B.M.Patil 
Medical College, Hospital and Research centre, Vijayapur. Ethical clearance was obtained from institutional ethical committee.

Two smears were collected from each subject, one smear was fixed in ethanol and other will be fixed in $20 \%$ commercially available honey (Two parts of honey+eight parts of distilled water). Smears were fixed in each fixative i.e ethanol and $20 \%$ honey for a minimum of 15 minutes. After which they were washed in tap water for $30 \mathrm{sec}$ and subjected to conventional Papanicolaou staining procedure. Smears were evaluated by following criteria (Table 1)

Data analysis:- Data was analyzed using 1. Mean \pm S.D, and 2 Chi square test

Inclusion criteria: All healthy individuals who visit for regular health check-up were included in the study.

\section{Exclusion criteria: Nil}

\section{Results}

A total of 200 cases were collected out of which 120 cases $(60 \%)$ were male and 80 cases $(40 \%)$ were female. Honey fixed smears (HF) showed acceptable overall cellularity and results of all cellular parameters were very much satisfactory and as good as ethanol fixed (EF) smears.

Out of 200 cases 193 (96.5) cases EF and 186 (93\%) cases of HF slides showed acceptable nuclear staining and 7 (3.5) cases of EF and 14 (7\%) cases of HF slides showed unacceptable nuclear staining which was statistically significant with $\mathrm{p}$ value of 0.008 . Similarly acceptable cytoplasmic staining was seen in $178(89 \%)$ cases of EF and $160(80 \%)$ cases of HF slides and $22(11 \%)$ cases of EF and $40(20 \%)$ cases of HF slides showed unacceptable cytoplasmic staining which showed no statistical difference between both fixatives with $p$ value of 0.821 . (Table 2)

Well preserved cell morphology was noted in 181 (90.5\%) cases of EF and 188 (94\%) cases of HF slides which showed no statistical difference between both fixatives with $\mathrm{p}$ value of 0.092 . Clarity of staining and uniformity of staining was present in $190(95 \%)$ cases EF, $176(88 \%)$ cases of HF slides and $191(95.5 \%)$ cases EF and $184(92 \%)$ cases of HF slides respectively which was statistically significant with $p$ value of $<0.005$. HF smears revaluated after a period of 6 months showed unchanged cellular parameters as described above in comparison to EF smears. (Table 2)

Table 1: Evaluation criteria.

\begin{tabular}{|c|c|c|}
\hline Features & Scores and criteria & Scores and criteria \\
\hline Nuclear staining & $\begin{array}{l}\text { Acceptable }=1 \\
\text { Round, smooth and clear nuclear } \\
\text { membrane }\end{array}$ & $\begin{array}{l}\text { Unacceptable }=0 \\
\text { Granular, disintegrated and out of focus }\end{array}$ \\
\hline Cytoplasmic staining & $\begin{array}{l}\text { Acceptable }=1 \\
\text { Intracytoplasmic membrane and } \\
\text { transparent cytoplasm }\end{array}$ & $\begin{array}{l}\text { Unacceptable }=0 \\
\text { Disintegrated cytoplasmic membrane, } \\
\text { granular cytoplasm and out of focus }\end{array}$ \\
\hline Cell morphology & $\begin{array}{l}\text { Preserved }=1 \\
\text { Absence of folds, no overlap and } \\
\text { maintained nuclear to cytoplasmic ratio }\end{array}$ & $\begin{array}{l}\text { Unpreserved }=0 \\
\text { Over lapping cells, folded and } \\
\text { disintegrated cells }\end{array}$ \\
\hline Clarity of staining & $\begin{array}{l}\text { Present }=1 \\
\text { Crispness in staining and transparency }\end{array}$ & $\begin{array}{l}\text { Absent }=0 \\
\text { Obliterate the nucleus and cytoplasm }\end{array}$ \\
\hline Uniformity of staining & $\begin{array}{l}\text { Present }=1 \\
\text { Uniformly stained throughout the } \\
\text { individual cell }\end{array}$ & $\begin{array}{l}\text { Absent }=0 \\
\text { Stained in different shades of color in } \\
\text { an individual cell }\end{array}$ \\
\hline
\end{tabular}

Table2: Distribution of cases comparing various cytomorphological features of Ethanol fixed smears and Honey fixed smears.

\begin{tabular}{|c|c|c|c|c|c|c|}
\hline \multirow{2}{*}{ Staining } & \multirow{2}{*}{ Scale } & \multicolumn{2}{|c|}{ Ethanol fixed } & \multicolumn{2}{|c|}{ Honey fixed } & \multirow{2}{*}{$p$ value } \\
\hline & & $\mathbf{N}$ & $\%$ & $\mathbf{N}$ & $\%$ & \\
\hline \multirow{2}{*}{ Nuclear staining } & Unacceptable & 7 & 3.5 & 14 & 7 & \multirow{2}{*}{$0.008^{*}$} \\
\hline & Acceptable & 193 & 96.5 & 186 & 93 & \\
\hline \multirow{2}{*}{ Cytoplasmic staining } & Unacceptable & 22 & 11 & 40 & 20 & \multirow{2}{*}{0.821} \\
\hline & Acceptable & 178 & 89 & 160 & 80 & \\
\hline
\end{tabular}




\begin{tabular}{|c|c|c|c|c|c|c|}
\hline \multirow{2}{*}{ Staining } & \multirow{2}{*}{ Scale } & \multicolumn{2}{|c|}{ Ethanol fixed } & \multicolumn{2}{|c|}{ Honey fixed } & \multirow{2}{*}{$\mathrm{p}$ value } \\
\hline & & $\mathbf{N}$ & $\%$ & $\mathbf{N}$ & $\%$ & \\
\hline \multirow{2}{*}{ Cell morphology } & Unpreserved & 19 & 9.5 & 12 & 6 & \multirow{2}{*}{0.092} \\
\hline & Preserved & 181 & 90.5 & 188 & 94 & \\
\hline \multirow{2}{*}{ Clarity of staining } & Absent & 10 & 5 & 24 & 12 & \multirow{2}{*}{$0.005^{*}$} \\
\hline & Present & 190 & 95 & 176 & 88 & \\
\hline \multirow{2}{*}{ Uniformity of staining } & Absent & 9 & 4.5 & 16 & 8 & \multirow{2}{*}{$<0.001^{*}$} \\
\hline & Present & 191 & 95.5 & 184 & 92 & \\
\hline \multicolumn{2}{|l|}{ Total } & 200 & 100 & 200 & 100 & \\
\hline
\end{tabular}

Note: *significantly associated at $5 \%$ level of significance

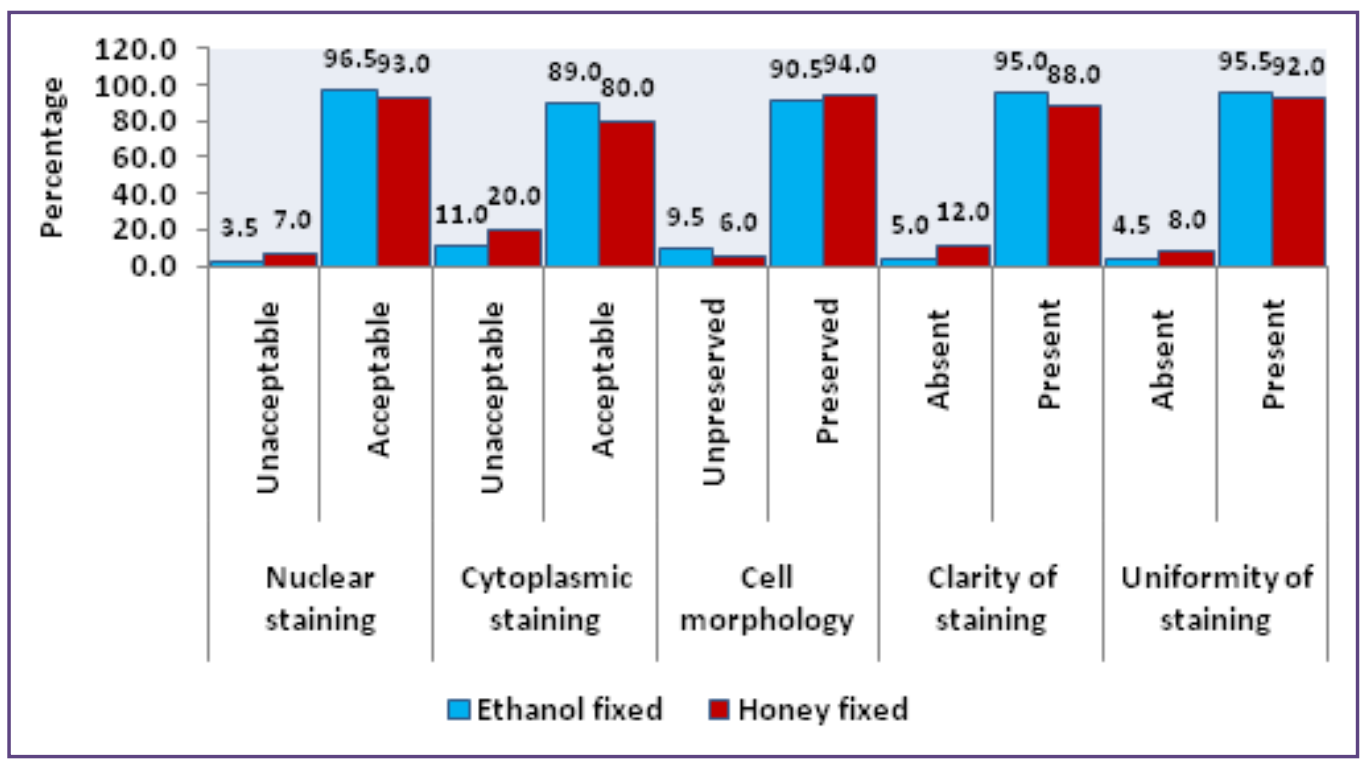

Fig. 1: Distribution of percentage of acceptable and unacceptable honey and ethanol fixed smears.

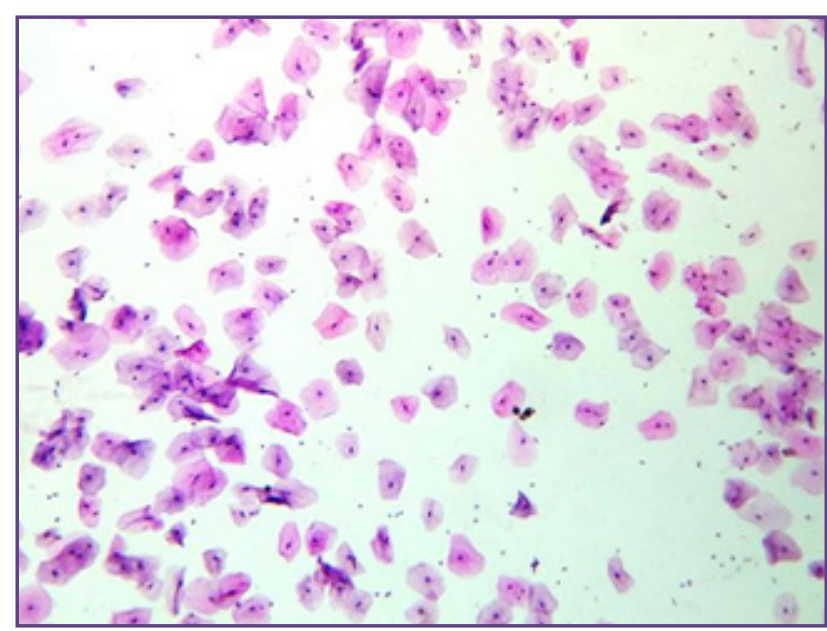

Fig. 2a: Ethanol fixed smear showing adequate cellularity, acceptable nuclear and cytoplasmic staining. - PAP 100X.

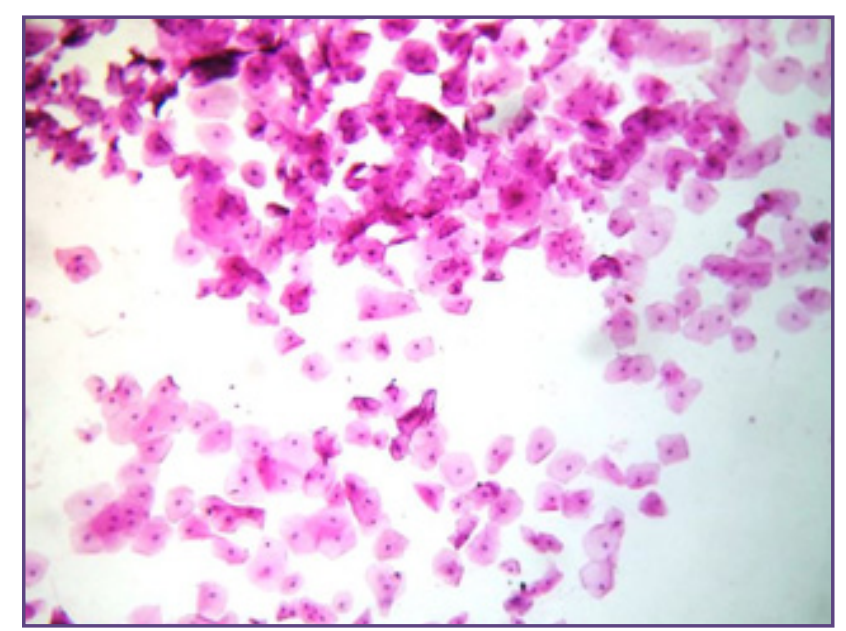

Fig. 2B: Honey fixed smear showing adequate cellularity, acceptable nuclear and cytoplasmic staining. - PAP 100X. 


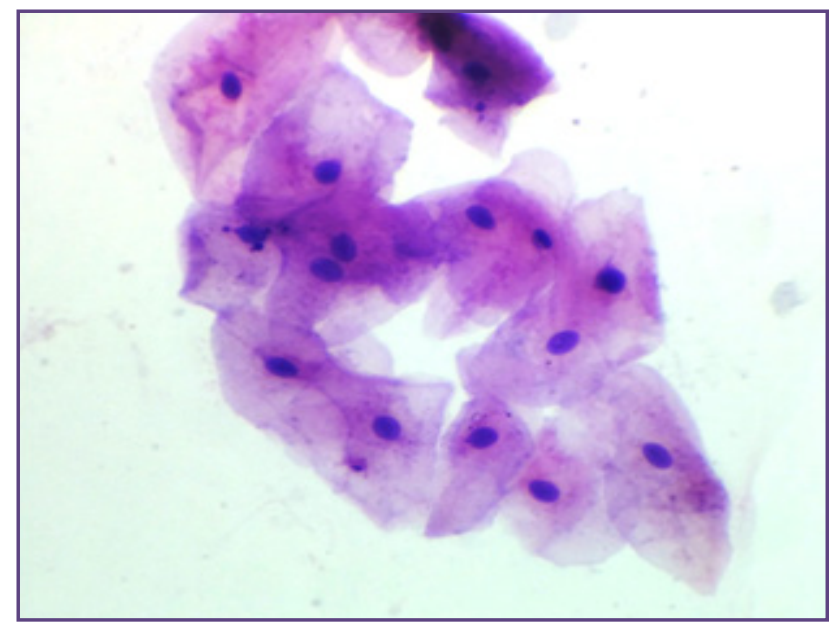

Figure 3A: Ethanol fixed smear showing preserved cell morphology, acceptable uniformity and clarity of staining nuclear staining - PAP 400X.

\section{Discussion}

Group of cells which are building blocks of living organism unite to form a tissue which perform specific function. Microscopic study of individual cell in a smear is called cytology and study of tissue is called histology. Fixation preserves the cells similar to living state, when these are subjected to staining aids in cytological examination and diagnosis. Though many fixatives are used in both cytology and histology, each of them has certain advantages and disadvantages. Ethanol is a gold standard fixative widely used as cytological fixative in many laboratories. Advantages are rapid fixation, antibacterial properties and acceptable preservation of cytological details, but major disadvantage being not freely available, costly and inflammable which prevents it from being an ideal fixative. ${ }^{6]}$ So in search of an ideal fixative honey could be a natural, cheap and safe alternative to ethanol as it has all inherent properties which are required for fixation due to its low $\mathrm{pH}$, high osmolarity and antibacterial properties. ${ }^{[7,8]}$

Many different studies have already been done to compare honey as fixative in histopatholgy in comparison to formalin, which has provided convincing and appreciable results. ${ }^{[9-11]}$ In honey fixed smears one could very clearly appreciate all cellular details such as nuclear, cytoplasmic staining, cellular morphology, clarity and uniformity of the staining which are almost equivalent to ethanol fixed smears. Present study in concordance with Singh A, et al ${ }^{[4]}$ showed that cellularity and cell morphology were well preserved in honey which provides adequate cytological material for diagnosis.

In present study comparison of ethanol and honey fixed smears for nuclear staining ( $p$ value $=0.008$ ), clarity of staining $(p$ value $=0.005)$, uniformity of staining ( $p$ value

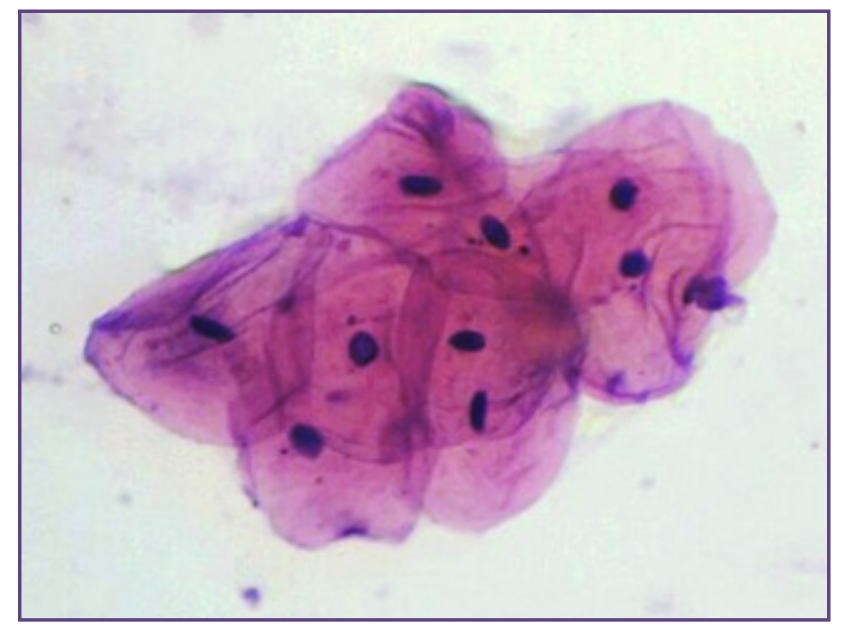

Figure 3B: Honey fixed smear showing acceptable preserved cell morphology, acceptable uniformity and clarity of staining nuclear staining PAP 400X.

$<0.001)$ were statistically significant. This is in discordance with Singh A, et $a l^{4}$ and Ishaq $\mathrm{R}$ et $a l^{[12]}$ study in which nuclear staining, clarity of staining, uniformity of staining showed no statistical difference between both fixatives.

In present study comparison of ethanol and honey fixed smears for cytoplasmic staining $(\mathrm{p}$ value $=0.821)$, preservation of cell morphology ( $p$ value $=0.092$ ) showed no statistical difference between both fixatives. This is in accordance with Singh A, et $a l^{[4]}$ and Ishaq $\mathrm{R}$ et al $^{[12]}$ study in which nuclear staining, clarity of staining, uniformity of staining also showed no statistical difference between both fixatives. In present study different routine cytological smears from malignant lesion, lymphnode, necrotizing lesions etc were not studied, hence outcome of cellular fixation and cellular details in such honey fixed smears needs to be studied in detail.

Similar studies have also been done to compare fixative ability of honey in comparison to formalin in histopathology. Ozakan $\mathrm{N}$ et al ${ }^{[2]}$ study which compared honey with neutral buffered formalin and alcohol formalin various lesion in histopathology. Nuclear morphology showed no statistically significant difference between alcoholic formalin $(3.25 \pm 0.13)$ and honey $(2.83 \pm 0.2)$ fixation $(\mathrm{p}>0.05)$. Similarly there was no significant difference among these fixatives with regard to cytoplasmic detail $(p>0.05)$ Even immunohistochemical comparison done in Ozakan $\mathrm{N}$ et al ${ }^{[2]}$ study for honey fixed and formalin fixed paraffin embedded tissue with Vimentin and Ki67 showed convincing results. There were no statistically significant differences among the various fixatives compared. $(p>0.05)$ The present study showed that honey fixed smears showed almost similar results when compared to ethanol fixed smears. Background 
of honey fixed slides was clear as comparable to ethanol fixed slides and most of the cells showed well defined nuclear chromatin, nuclear membrane and intact cytoplasm. Even Immunohistochemistry could be done on honey fixed slides as it fixes tissue without damaging or altering the antigens present in the tissues. ${ }^{[13,14]}$

\section{Conclusion}

Honey being a natural, economical and pleasant smelling, easily available, eco-friendly innovative fixative with antibacterial properties. Honey as fixative has shown cytomorphological features comparable to ethanol. Using honey also improves the safety and work environment in the laboratory. In rural areas, health camps, public health service centres and in absence of alcohol fixatives, honey can be used as a successful alternative.

\section{References}

1. Rhodes A. Fixation of tissues. In: Suvarna KS, Layton C, Bancroft DJ editors. Bancroft's Theory and Practice of Histological Techniques. 7th ed. Churchill Livingstone: Elsevier; 2013.p69-93.

2. Özkan N, Şalva E, Cakalağaoğlu F, Tüzüner B. Honey as a substitute for formalin?. Biotechnic \& Histochemistry. 2012 Feb 1;87(2):148-53

3. Culling CFA, Allison RT, Barr WT. Fixation, processing, microtomy. In:Culling CFA Editors. Cellular Pathology Technique. 4th ed. London:Butterworths;p35

4. Singh A, Hunasgi S, Koneru A, Vanishree M, Ramalu S, Manvikar V. Comparison of honey with ethanol as an oral cytological fixative: A pilot study. Journal of cytology.2015 Apr;32(2):113.

5. Patil S, Premalatha BR, Rao RS, Ganavi BS. Revelation in the field of tissue preservation-A preliminary study on natural formalin substitutes. Journal of international oral health: JIOH. 2013 Feb;5(1):31

6. Sona M, Preethamol S. A comparative study on the efficacy of honey and ethanol as cytological fixatives. International Journal of Advances in Medicine. 2017 May 23;4(3):762-7.

7. Bizerra FC, Da Silva Jr PI, Hayashi MA. Exploring the antibacterial properties of honey and its potential. Frontiers in microbiology. 2012:3:398.

8. McCarthy J. antibacterial effects of honey: medical fact or fiction?. American Bee Journal. 1995:171-2.

9. Sabarinath B, Sivapathasundharam B, Sathyakumar M. Fixative properties of honey in comparison with formalin. Journal of Histotechnology. 2014 Mar1;37(1):21-5.

10. Muddana K, Muppala JN, Dorankula SP, Maloth AK, Kulkarni PG, Thadudari D. Honey and olive oil as biofriendly substitutes for formalin and xylene in routine histopathology. Indian Journal of Dental Research. 2017 May1;28(3):286.

11. Lalwani V, Surekha R, Vanishree M, Koneru A, Hunasgi S, Ravikumar S. Honey as an alternative fixative for oral tissue: An evaluation of processed and unprocessed honey. Journal of oral and maxillofacial pathology. 2015 Sep;19(3):342.

12. Ishaq R, Azmat H, Omair M, Sheikh AK, Tanwani AK. Comparison Of Honey With Alcohol As A Fixative In Fine Needle Aspiration Cytology. International Journal of Pathology. 2017;15(1):15-8.

13. Majumdar B, Rao R, Patil S. Tissue Preservation with Natural Fixatives: An Immunohistochemical Evaluation. World Journal of Dentistry. 2016;7:87-91

14. Gunter M, Bryant P. Immunocytochemical evaluation of ductal carcinoma in breast after preservation in honey. Journal of Histotechnology. 2009 Jun 1;32(2):54-9.

*Corresponding author:

Dr Mahmood Nawaz Khan, Room No 112, PG 2 Hostel, Shri B.M Patil Medical College, Hospital and Research Centre. B.L.D.E University,

Vijayapura 586103, Karnataka, India

Phone: +918801566948

Email: drnawazkhan0@gmail.com

Financial or other Competing Interests: None. 